\title{
ИНТЕГРИРОВАННЫЙ ПОДХОД К АНАЛИЗУ \\ СМЕРТНОСТИ ПО ПРИЧИНАМ СМЕРТИ: ТАБЛИЦЫ \\ СМЕРТНОСТИ ПРИ УСЛОВИИ УСТРАНЕНИЯ \\ ОТДЕЛЬНЫХ ПРИЧИН СМЕРТИ И ДЕКОМПОЗИЦИЯ \\ ОЖИДАЕМОЙ ПРОДОЛЖИТЕЛЬНОСТИ ЖИЗНИ
}

\author{
ХИРАМ БЕЛТРАН-САНЧЕС, САМУЭЛЬ Х. ПРЕСТОН, \\ ВЛАДИМИР КАНУДАС-РОМО
}

\begin{abstract}
Статья объединяет два метода анализа влияния причин смерти на ожидаемую продолжительность жизни. Один оценивает вклад в ее изменение различных причин смерти; другой анализирует влияние на продолжительность жизни устранения смертей от отдельной причины. Новые формуль позволяют объединить оба метода и делают более понятным взаимодействие причин смерти в их влиянии на ожидаемую продолжительность жизни. Мы применили наш подход при анализе изменения продолжительности жизни населения США между 1970 и 2000 г2. Mbl демонстрируем и объясняем тот парадокс, что, несмотря на снижение смертности от рака, приведшее к увеличению продолжительности жизни между 1970 и 2000 г2., в 2000 г. рак был ответственен за большее число потерянных лет жизни, чем в 1970 г.
\end{abstract}

Ключевые слова: таблицы смертности, декомпозиция продолжительности жизни, причинь смерти, таблииь смертности при условии устранения отдельных причин смерти, США.

\section{ВВЕДЕНИЕ}

Ожидаемая продолжительность жизни при рождении, вычисляемая для определенного периода, представляет собой обобщенную характеристику условий смертности, наблюдаемой в этот период. Оценка роли разных причин смерти, влияющих на ожидаемую продолжительности жизни и её изменения, - область активных демографических исследований. Обычно используются два научных подхода: 1) оценка продолжительности жизни при условии исключения данной причины и 2) оценка вклада разных причин смерти в различия или изменения продолжительности жизни. Первый подход рассматривает только одно население и ведет к развитию таблиц смертности с единственным выбытием (часто - «таблицы смертности при условии устранения отдельных причин смерти»). Второй подход, сравнительный, привел к развитию методов декомпозиции, с помощью которых определяют ответственность за различия смертности конкретных причин смерти.

\footnotetext{
ХИРАМ БЕЛТРАН-САНЧЕС (beltrans@ucla.edu), ШКОЛА ОБЩЕСТВЕННОГО ЗДОРОВЬЯ КАЛИФОРНИЙСКОГО УНИВЕРСИТЕТА В ЛОС-АНДЖЕЛЕСЕ, США.
}

САМУЭЛЬ Х. ПРЕСТОН, УНИВЕРСИТЕТ ПЕНСИЛЬВАНИИ, США.

ВЛАДИМИР КАНУДАС-РОМО, АВСТРАЛИЙСКИЙ НАЦИОНАЛЬНЫЙ УНИВЕРСИТЕТ, АВСТРАЛИЯ.

ПЕРЕВОД ОРИГИНАЛЬНОЙ СТАТЬИ: BELTRAN-SANCHEZ H., S.H. PRESTON, V. CANUdAS-Romo (2008). AN INTEgRATED APPROACH TO CAUSE-OF-DEATH ANALYSIS: CAUSE-DELETED LIFE TABLES AND DECOMPOSITIONS OF LIFE EXPECTANCY // DEMOGRAPHIC RESEARCH. 19 (35): 1323-135O. DOI: HTTPS://DX.DOI.ORG/10.4054/DEMRES.2008.19.35 
Эти подходы - родственные, но их связь между собой никогда не была продемонстрирована. В данной статье мы предлагаем новые формулы декомпозиции и показываем, что они непосредственно связаны с формулами, описывающими таблицы смертности при устранении некоторых причин смерти. Мы используем предлагаемый подход для анализа данных по США за 1970 и 2000 гг.

\section{1. ИСХОДНЫЕ ПОЛОЖЕНИЯ}

Хотя демографы давно используют таблицы смертности при анализе смертности, разработка таблиц смертности, в которых отражался бы вклад отдельных причин смерти, началась гораздо позднее [Brownlee 1919; Fisher, Vigfusson, Dickson 1922; Pearl 1922; Greville 1948; Jordan 1952; Chiang 1968; Spiegelman 1968, Preston, Keyfitz, Schoen 1972]. Первая официальная таблица смертности по причинам смерти в США за десятилетний период была опубликована в конце 1960-х гг. [United States... 1968].

Один из самых важных результатов использования таких таблиц - оценка увеличения ожидаемой продолжительности жизни при рождении при условии устранения какой-либо причины смерти, т.е. при условии, что коэффициент смертности от этой причины принимается равным нулю, а коэффициенты смертности от всех других причин остаются неизменными. Чтобы дать общее представление о математике таких вычислений, предположим, что имеется $n$ взаимоисключающих причин смерти в населении в период времени $t$. Вероятность дожить от момента рождения до возраста $a$ в период времени $t$, если существует единственная причина смерти $i$, равна:

$$
p_{i}(a, t)=e^{-\int_{0}^{a} \mu_{i}(s, t) d s},
$$

где $\mu_{i}(s, t)$ - коэффициент смертности от причины $i$ в возрастном интервале от $s$ до $s+d s . \mathrm{B}$ общих таблицах смертности эквивалентная функция дожития равна:

$$
p_{i}(a, t)=e^{-\int_{0}^{a} \mu_{i}(s, t) d s}, \text { где } \mu(s, t)=\sum_{i=1}^{n} \mu_{i}(s, t) .
$$

Для простоты, пусть $p(a, t)=p(a)$.

Если предположить, что $n$ причин смерти независимы, то $p(a)=p_{1}(a) \cdot p_{2}(a) \cdot \ldots \cdot p_{n}(a)$.

Пусть $p_{-i}(a)=\frac{p(a)}{p_{i}(a)}-$ вероятность дожития в возрасте $a$ от всех причин за исключением причины $i$. Если известна сила смертности, $\mu(s)$, то ожидаемая продолжительность жизни при рождении вычисляется как:

$$
e(0)=\int_{0}^{\omega} e^{-\int_{0}^{a} \mu(s) d s} d a=\int_{0}^{\omega} p(a) d a .
$$

Если существуют $n$ взаимоисключающих и исчерпывающих причин смерти, то продолжительность жизни вычисляется как: 


$$
e(0)=\int_{0}^{\omega} p_{1}(a) p_{2}(a) \ldots p_{n}(a) d a
$$

Пусть $D_{i}(0)$ - годы жизни, дополнительно выигранные при рождении, если устранена причина смерти $i$ :

$$
D_{i}(0)=\int_{0}^{\omega} p_{i}(a) d a-\int_{0}^{\omega} p_{-i}(a) p_{i}(a) d a
$$

Важно отметить, что расчеты, выполненные при условии устранения некоторых причин смерти, лучше интерпретировать как упражнение в вычислениях, а не как эпидемиологический прогноз. Поскольку причины смерти являются взаимоисключающими, а их перечень - исчерпывающим, то коэффициенты смертности от отдельных причин в сумме равны коэффициенту смертности от всех причин. Эта аддитивность делает данное соотношение аналогичным описанию изменения численности населения с помощью уравнения демографического баланса. Влияние смертности от определенной причины на показатели таблиц смертности можно оценить путем построения таблицы смертности без учета данной причины. Результаты расчета позволяют оценить, как интенсивность и возрастное распределение смертей от рассматриваемой причины влияют на все показатели таблицы смертности, включая ожидаемую продолжительность жизни. Чтобы использовать такой подход для эпидемиологического прогноза, необходимо допустить, что все смерти от рассматриваемой причины устранимы, что возможно крайне редко, и что коэффициенты смертности от других причин не изменятся при устранении одной из них. Подобное допущение может быть уместным в качестве первого приближения для больших групп причин смерти, таких как инфекционные заболевания, насильственные смерти, сердечно-сосудистые заболевания и рак, но оно едва ли оправдано применительно к таким заболеваниям как диабет, который может являться сопутствующим заболеванием при разных причинах смерти.

\section{2. ПОДХОДЫ К ДЕКОМПОЗИЦИИ}

В начале 1980-х гг. методика декомпозиции изменений ожидаемой продолжительности жизни по возрасту и причинам смерти была независимо предложена Арриагой [Arriaga 1982; 1984], Андреевым [1982], Поллардом [Pollard 1982] и Пресcа [Pressat 1985]. Предложенные методики математически схожи [Pollard 1988], хотя, как показано ниже, их применение с использованием дискретных приближений может привести к разным результатам. Дальнейшее развитие метода позволило применять декомпозицию к другим демографическим измерителям и привнесло динамические элементы [Andreev et al. 2002; Vaupel, Canudas-Romo 2002; Vaupel, Canudas-Romo 2003].

Первоначальная цель и главный фокус декомпозиции были сосредоточены не на причинах смерти, а на возрасте. Декомпозиция изменений и различий смертности по возрасту предшествовала декомпозиции смертности по причинам смерти, последняя развивалась вслед за первой. Если бы первоначальной целью была декомпозиция по 
причинам, то был бы доступен более точный понятийный набор, который разработан только сейчас.

Как показано выше, если в населении действует $n$ взаимоисключающих и исчерпывающих причин смерти, то $p(a)=p_{1}(a) \cdot p_{2}(a) \cdot \ldots \cdot p_{n}(a)$. Если предположить, что сила смертности ( $\mu(a))$ дифференцируема по времени, то изменение вероятности дожить от момента рождения до возраста $a$ в зависимости от времени можно записать в следующем виде:

$$
\dot{p}(a)=\sum_{i=1}^{n} \dot{p}_{i}(a) p_{-i}(a)
$$

где точка над переменной означает производную по времени. Таким образом, непрерывное изменение ожидаемой продолжительности жизни при рождении может быть представлено как: ${ }^{1}$

$$
\dot{e}(0)=\sum_{i=1}^{n} \int_{0}^{\omega} \dot{p}_{i}(a) p_{-i}(a) d a .
$$

Формула (2) - непосредственное выражение декомпозиции изменений ожидаемой продолжительности жизни по причинам смерти. Она четко показывает, что вклад каждой причины в изменение продолжительности жизни зависит от изменений выживаемости от конкретной причины (причина $i$ ), взвешенной по накопленной вероятности выжить от оставшихся причин (причина -i). Интеграл в формуле (2) может быть разбит по возрастным группам для того, чтобы оценить вклад в изменение ожидаемой продолжительности жизни каждой возрастной группы. Таким образом, уравнение (2) может оценивать как вклад возрастных групп, так и отдельных причин смерти в изменение продолжительности жизни $e(0)$. Как показано ниже, уравнение (2) - основополагающее для объединения метода декомпозиции и метода построения таблиц смертности при условии устранения причин смерти.

Для дискретных временных интервалов примем, что $e(0)$ и $e^{*}(0)$ - ожидаемая продолжительность жизни при рождении в моменты времени 1 и 2 соответственно. Тогда (см. подробнее Приложение 1 в статье [Beltrán-Sánchez, Preston 2007]):

$$
e^{*}(0)-e(0) \cong \sum_{i=1}^{n} \int_{0}^{\infty}\left(p_{i}^{*}-p_{i}\right)\left(\frac{p_{-i}+p_{-i}^{*}}{2}\right) d a .
$$

Для дискретных возрастных интервалов приведенная выше формула эквивалентна выражению:

$$
e^{*}(0)-e(0)=\sum_{i=1}^{n} \sum_{x=0,5}^{\omega}\left({ }_{n} L_{x, i}^{*}-{ }_{n} L_{x, i}\right)\left(\frac{{ }_{n} L_{x,-i}^{*}+{ }_{n} L_{x,-i}}{2 n}\right)
$$

где ${ }_{n} L_{x, i},{ }_{n} L_{x,-i},{ }_{n} L_{x, i, n}^{*} L_{x,-i}^{*}-$ число человеко-лет, прожитых в возрастах от $x$ до $x+n$ к

\footnotetext{
1Уравнение (2) также может быть получено из уравнения(36) Вопеля и Канудас-Ромо [2003:209].
} 
моментам времени 1 и 2 в таблицах смертности для причины $i$ и причины $-i$ соответственно, при корне таблицы смертности, равном 1 (см. подробнее Приложение 3).

В таблице П1 Приложения сравниваются результаты, полученные при нашем подходе к декомпозиции по причинам смерти и при подходах, предлагаемых Поллардом и Арриагой. Наш подход и подход Полларда дают близкие результаты. Подход Арриаги может привести к недооценке вклада основных причин смерти, сконцентрированных преимущественно в старших возрастах.

\section{4. СВЯЗЬ МЕЖДУ МЕТОДОМ ДЕКОМПОЗИЦИИ И ТАБЛИЦАМИ СМЕРТНОСТИ ПРИ УСЛОВИИ УСТРАНЕНИЯ ПРИЧИН СМЕРТИ}

Как показано в уравнении (1), выигрыш в ожидаемой продолжительности жизни при рождении в момент времени $t$ при устранении причины смерти $i$ вычисляется следующим образом:

$$
D_{i}(0)=\int_{0}^{\omega} p_{-i}(a) d a-\int_{0}^{\omega} p_{-i}(a) p_{i}(a) d a
$$

Соответственно, изменение $D_{i}(0)$ во времени выражается как:

$$
\dot{D}_{i}(0)=\int_{0}^{\omega} \dot{p}_{-i}(a) q_{i}(a) d a-\int_{0}^{\omega} \dot{p}_{i}(a) p_{-i}(a) d a,
$$

где $q_{i}(a)=1-p_{i}(a)$ и точка над переменной обозначает производную по времени.

Для дискретных временных интервалов пусть $D_{i}(0)$ и $D_{i}{ }^{*}(0)$ - выигрыш в ожидаемой продолжительности жизни при рождении в моменты времени 1 и 2 соответственно, благодаря устранению причины смерти $i$. Тогда, формула (3) может быть записана как (см. приложение 2):

$$
D_{i}^{*}(0)-D_{i}(0)=\int_{0}^{\infty}\left(p_{-i}^{*}-p_{-i}\right)\left(\frac{q_{i}+q_{i}^{*}}{2}\right) d a-\int_{0}^{\infty}\left(p_{i}^{*}-p_{i}\right)\left(\frac{p_{-i}+p_{-i}^{*}}{2}\right) d a,
$$

где $q_{i}=1-p_{i}$.

Для дискретных возрастных интервалов приведенная выше формула эквивалентна:

$$
\begin{aligned}
D_{i}^{*}(0)-D_{i}(0)= & \sum_{x=0,5}^{\omega}\left({ }_{n} L_{x,-i}^{*}-{ }_{n} L_{x,-i}\right)\left(1-\frac{{ }_{n} L_{x, i}+{ }_{n} L_{x, i}^{*}}{2 n}\right) \\
& -\sum_{x=0,5}^{\omega}\left({ }_{n} L_{x, i}^{*}-{ }_{n} L_{x, i}\right)\left(\frac{{ }_{n} L_{x,-i}+{ }_{n} L_{x,-i}^{*}}{2 n}\right) .
\end{aligned}
$$

Таким образом, изменение количества потерянных лет вследствие смертности от определенной причины смерти является функцией двух слагаемых. Второе слагаемое в 
уравнении (3) - изменение дожития умерших от причины $i$, взвешенное по кумулятивной вероятности дожития от причины $-i$. Из уравнения (2) четко следует, что изменение ожидаемой продолжительности жизни при рождении определяется изменением смертности от причины $i$. Таким образом, изменение в ожидаемой продолжительности жизни при условии устранения причины смерти $i$ тесно связано с изменением продолжительности жизни, обусловленным причиной смерти $i$ в формулах декомпозиции. Фактически, и то, и другое было бы абсолютно одинаковым, если бы смертность от других причин смерти оставалась неизменной, поскольку в таком случае $\dot{p}_{-i}(a)$ в первом слагаемом было бы равно нулю. Другими словами, положительный вклад причины смерти в изменение ожидаемой продолжительности жизни был бы абсолютно равен сокращению потерянных лет жизни от данной причины (отсюда знак «-» перед вторым членом уравнения (3)). В общем виде, первое слагаемое в уравнении (3) показывает изменение дожития от причины $-i$, взвешенной по кумулятивной вероятности умереть от причины $i$. Его абсолютное значение может быть большим лишь в том случае, если причина $i$ является одной из

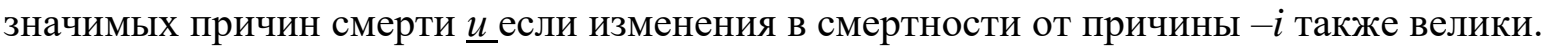

Чтобы проиллюстрировать смысл уравнения (3), предвосхитим результат, который будет получен в дальнейшем. Предположим, что мы изучаем изменение смертности по причинам смерти, включая смертность от рака, между двумя моментами времени. Предположим далее, что снижение смертности от рака приведет к увеличению продолжительности жизни за данный период (второе слагаемое в уравнении (3) для рака отрицательно). Теперь предположим, что мы построили таблицы смертности при условии устранения причин смерти между моментами времени 1 и 2. Второе слагаемое в уравнении (3) привело бы к предположению, что левая часть уравнения (3) должна быть отрицательной: смертность от рака должна бы вызывать меньшие потери продолжительности жизни в конечный момент времени по сравнению с начальным. Тем не менее, если смертность от других причин смерти значительно сокращается за данный период (т.е. первое слагаемое в уравнении (3) имеет положительный знак), смертность от рака в действительности может вызывать большие потери продолжительности жизни в момент времени 2. Очевидно, что изменение вклада смертности от рака в сокращение продолжительности жизни зависит от изменения смертности от других причин, как показано в уравнении (3).

\section{5. ПРИМЕР НЕДАВНИХ ИЗМЕНЕНИЙ СМЕРТНОСТИ В США}

\section{1 Декомпозиция изменений по причинам смерти}

После нескольких десятилетий медленных улучшений в смертности, в период с 1970 по 2000 г. в США были достигнуты большие успехи в увеличении продолжительности жизни. Общепризнано, что в основном это стало результатом снижения смертности от болезней системы кровообращения, обусловленного медицинскими инновациями, такими как аортокоронарное шунтирование, широкое применение препаратов, снижающих артериальное давление, статинов, бета-блокаторов, а также снижением курения [Cutler 2004; Ergin et al. 2004; Ford et al. 2007]. 
Данные по смертности за 1970 г. взяты из общедоступного массива данных о множественных причинах смерти за 1968-1973 гг., созданного в рамках Межуниверситетского консорциума политических и социальных исследований [Interuniversity Consortium...2004]. За 2000 г. мы используем общедоступный массив данных о множественных причинах смерти, опубликованный Национальным центром статистики здравоохранения [National Center...2002]. Среднегодовая численность населения за 1970 и 2000 гг. взята из данных Бюро цензов [U.S. Bureau of Census 1971; U.S. Bureau of Census $2005]^{2}$. Для оценки численности черного населения в 1970 г. мы использовали данные из работы [Preston et al. 1998] ${ }^{3}$. Открытый возрастной интервал в данном исследовании составляет $100+{ }^{4}$. Мы рассматриваем 11 основных групп причин смерти в США в 2000 году и конструируем аналогичные категории для 1970 г. ${ }^{5}$ К ним относятся следующие группы причин: болезни сердца, цереброваскулярные заболевания, злокачественные новообразования, хронические заболевания нижних дыхательных путей; насильственные смерти (несчастные случаи, самоубийства и убийства); диабет; грипп и пневмония; нефрит (нефрит, нефротический синдром и нефроз); сепсис; цирроз печени (хронические заболевания печени и цирроз печени); гипертония (первичная артериальная гипертензия и гипертонические заболевания почек). Сопоставимые коды для 1970 г. получены из Центра по контролю заболеваний и профилактике [Centers for Disease Control 2001].

\subsection{1 Результаты}

Мы оцениваем ожидаемую продолжительность жизни при рождении для всего населения США в 1970 г. в 70,7 года (немного ниже значения в 70,9 года, полученного Национальным центром статистики здравоохранения [National Center...2002]) и в 76,96 года в 2000 г. (немного выше, чем оценки Ариаса [Arias 2002]). Таким образом, по нашим оценкам, в период с 1970 по 2000 г. ожидаемая продолжительность жизни при рождении для всего населения США увеличилась на 6,26 года. Соответствующие цифры в разбивке по расе и полу представлены в таблице 1.

На долю 11 групп причин смерти, отобранных для настоящего исследования, приходится основная часть смертей в оба рассматриваемых года. В 1970 г. примерно 84\% всех смертей относятся к одной из этих причин, в 2000 г. их доля сократилась до 80\% (Таблица 2). На долю болезней системы кровообращения (болезни сердца и цереброваскулярные заболевания) и злокачественных новообразований приходится $66 \%$ всех смертей в 1970 г. и 59\% в 2000 г.

\footnotetext{
2 Для 2000 г. мы использовали ежемесячные постпереписные оценки численности постоянного населения, доступные в электронном виде.

${ }^{3}$ Мы использовали оценки из [Preston et al. 1998] для возрастных групп 0-84 года и переписные оценки для возрастов 85-99 лет [U.S. Bureau of Census 1971].

4 Численность долгожителей (населения, достигшего возраста 100 лет и более) в 1970 г. была посчитана неверно [Siegel 1974]. Поэтому мы используем предпочтительные оценочные данные о численности долгожителей в разрезе расы и возраста, содержащиеся в работе [Siegel, Passel 1976].

5 Перечень основных причин смерти за 2000 г. определен на основе Сводной таблицы: Отчет об основных причинах смерти в 1999-2004 гг., опубликованной на сайте Центра по контролю заболеваний и профилактике [Centers for Disease Control 2005].
} 
Таблица 1. Изменение ожидаемой продолжительности жизни при рождении в США в 1970-2000 гг., по расе и полу

\begin{tabular}{l|c|c|c|c|c|c|c|c|c}
\hline \multirow{2}{*}{ Годы } & \multicolumn{3}{|c|}{ Все население } & \multicolumn{3}{c|}{ Белое население } & \multicolumn{3}{c}{ Черное население } \\
\cline { 2 - 9 } & $\begin{array}{c}\text { Оба } \\
\text { пола }\end{array}$ & $\begin{array}{c}\text { Муж- } \\
\text { чины }\end{array}$ & $\begin{array}{c}\text { Жен- } \\
\text { щины }\end{array}$ & $\begin{array}{c}\text { Оба } \\
\text { пола }\end{array}$ & $\begin{array}{c}\text { Муж- } \\
\text { чины }\end{array}$ & $\begin{array}{c}\text { Жен- } \\
\text { щины }\end{array}$ & $\begin{array}{c}\text { Оба } \\
\text { пола }\end{array}$ & $\begin{array}{c}\text { Муж- } \\
\text { чины }\end{array}$ & $\begin{array}{c}\text { Жен- } \\
\text { щины }\end{array}$ \\
\hline 2000 & 76,96 & 74,24 & 79,56 & 77,44 & 74,79 & 79,99 & 71,74 & 68,15 & 75,06 \\
1970 & 70,70 & 66,97 & 74,62 & 71,58 & 67,88 & 75,46 & 64,78 & 61,16 & 68,66 \\
Разница & 6,26 & 7,27 & 4,94 & 5,86 & 6,91 & 4,53 & 6,96 & 6,99 & 6,40 \\
\hline
\end{tabular}

Источник: Массив данных о множественных причинах смерти за 1968-1973 г2. и за 2000 г.; оченки Бюро иензов.

Таблица 2. Процентное распределение смертей по причинам, все население США, 1970-2000 гг.

\begin{tabular}{|c|c|c|}
\hline Причины смерти & 1970 & 2000 \\
\hline Болезни сердца & 38,28 & 29,58 \\
\hline Злокачественные новообразования & 17,21 & 23,00 \\
\hline Цереброваскулярные заболевания & 10,78 & 6,98 \\
\hline Насильственные смерти & 8,08 & 5,29 \\
\hline Хронические болезни нижних дыхательных путей & 1,61 & 5,07 \\
\hline Диабет & 1,99 & 2,88 \\
\hline Грипп и пневмония & 3,26 & 2,72 \\
\hline Нефрит, нефротический синдром и нефроз & 0,46 & 1,55 \\
\hline Сепсис & 0,18 & 1,30 \\
\hline Хронические заболевания печени и цирроз печени & 1,63 & 1,11 \\
\hline Гипертензия и гипертонические болезни почек & 0,43 & 0,75 \\
\hline Другие причины & 16,07 & 19,79 \\
\hline Всего & 100,00 & 100,00 \\
\hline
\end{tabular}

Источник: См. табл. 1.

В таблице 3 и на рисунке 1 представлены наши оценки вклада каждой причины смерти в изменение ожидаемой продолжительности жизни при рождении в разбивке по расе и по полу в 1970-2000 гг. ${ }^{6}$ Снижение смертности от болезней сердца внесло наибольший вклад в увеличение ожидаемой продолжительности жизни за 30-летний периода для всех групп населения за исключением черных мужчин, для которых снижение смертности от насильственных смертей добавило 1,94 года в общий рост продолжительности жизни, который составил 6,99 года. Этот исключительно большое снижение насильственной смертности среди мужского черного населения привело к большему увеличению продолжительности жизни в сравнении с другими группами населения США. Снижение смертности от рака внесло положительный вклад в рост

\footnotetext{
${ }^{6}$ Дискретная версия уравнения (2) содержит слагаемые, характеризующие взаимодействие разных причин смерти, как показано в приложении 1 работы Белтрана-Санчеса и Престона [Beltrán-Sánchez, Preston 2007]. Вклад каждого слагаемого в изменение ожидаемой продолжительности жизни при рождении может быть оценен двумя путями: посредством вычисления разницы между последними двумя строками в таблице 3 или с использования формул из работы Белтрана-Санчеса и Престона [Beltrán-Sánchez, Preston 2007]. Вклад слагаемых, характеризующих взаимодействие разных причин смерти, очень незначителен, о чем говорит небольшая разница между суммой по причинам и общим изменением ожидаемой продолжительности жизни в таблице 3. Для большинства причин смерти этот вклад в продолжительность жизни находится в пределах одной сотой года. Если не принимать во внимание ошибки округления, прямая оценка вклада взаимодействия между причинами, при использовании формулы Белтрана-Санчеса и Престона [Beltrán-Sánchez, Preston 2007], дает результаты, идентичные с итогами, косвенно полученными из таблицы 3 (данные не отображены).
} 
продолжительности жизни для всего и для белого населения, но не для черного населения. Негативный вклад вносил также диабет, но только для мужчин.

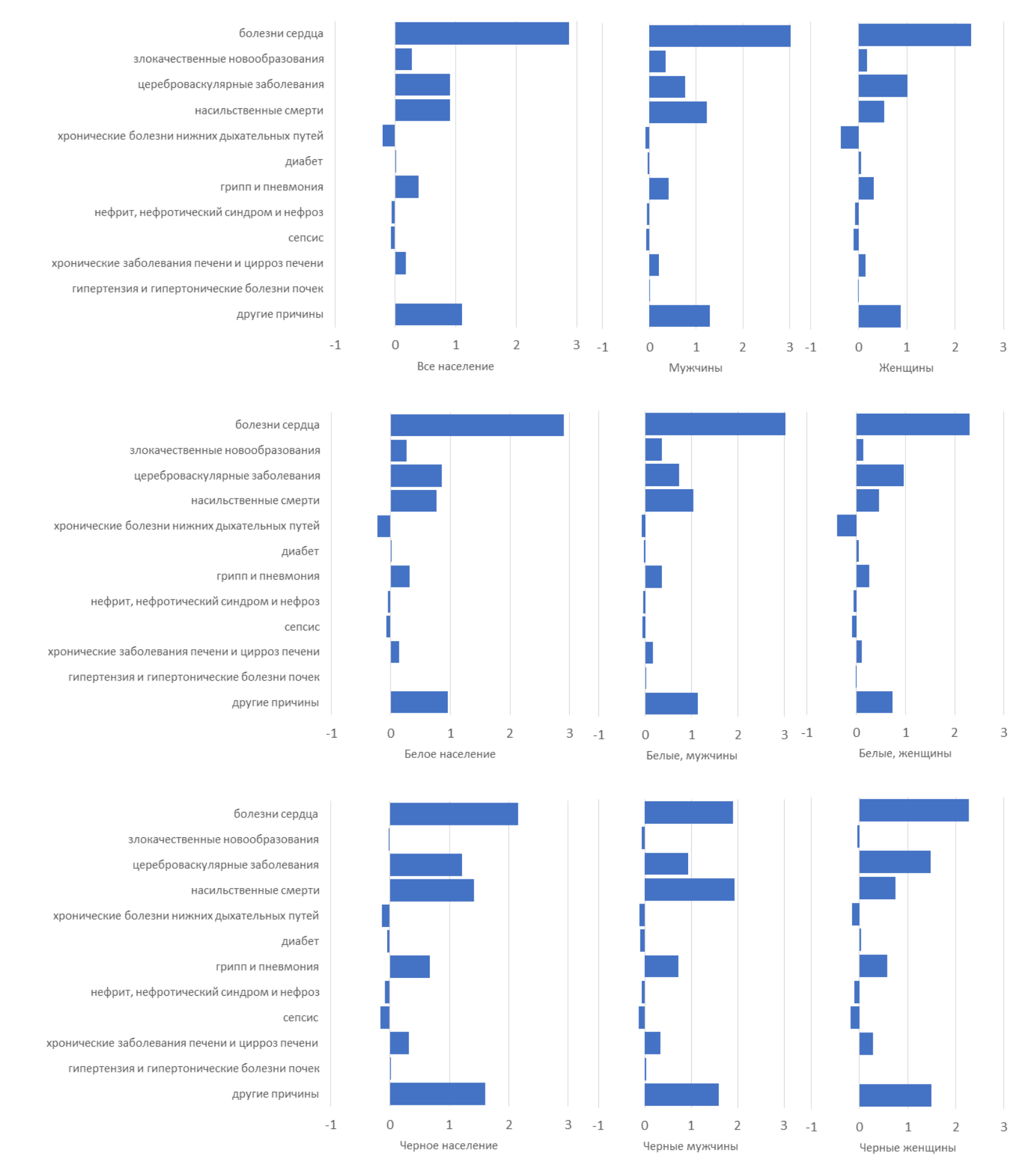

Рисунок 1. Вклад причин смерти в изменение ожидаемой продолжительности жизни при рождении в США, 1970-2000 гг., лет

Источник: См. табл. 3. 


\section{Таблица 3. Вклад различных причин смерти в изменение ожидаемой продолжительности жизни при рождении в США, 1970-2000 гг., лет}

\begin{tabular}{|c|c|c|c|c|c|c|c|c|c|}
\hline \multirow[b]{2}{*}{ Причины смерти } & \multicolumn{3}{|c|}{ Все население } & \multicolumn{3}{|c|}{ Белое население } & \multicolumn{3}{|c|}{ Черное население } \\
\hline & $\begin{array}{l}\text { Оба } \\
\text { пола }\end{array}$ & $\begin{array}{l}\text { Муж- } \\
\text { чины }\end{array}$ & $\begin{array}{l}\text { Жен- } \\
\text { щины }\end{array}$ & $\begin{array}{r}\text { Оба } \\
\text { пола } \\
\end{array}$ & $\begin{array}{l}\text { Муж- } \\
\text { чины } \\
\end{array}$ & $\begin{array}{l}\text { Жен- } \\
\text { щины }\end{array}$ & $\begin{array}{r}\text { Оба } \\
\text { пола }\end{array}$ & $\begin{array}{l}\text { Муж- } \\
\text { чины }\end{array}$ & $\begin{array}{l}\text { Жен- } \\
\text { щины }\end{array}$ \\
\hline Болезни сердца & 2,87 & 3,22 & 2,33 & 2,90 & 3,32 & 2,30 & 2,16 & 1,90 & 2,27 \\
\hline $\begin{array}{l}\text { Злокачественные } \\
\text { новообразования }\end{array}$ & 0,27 & 0,35 & 0,17 & 0,26 & 0,36 & 0,15 & $-0,02$ & $-0,06$ & $-0,04$ \\
\hline $\begin{array}{l}\text { Цереброваскулярные } \\
\text { заболевания }\end{array}$ & 0,90 & 0,77 & 1,02 & 0,85 & 0,73 & 0,96 & 1,21 & 0,94 & 1,48 \\
\hline $\begin{array}{l}\text { Насильственные } \\
\text { смерти }\end{array}$ & 0,90 & 1,23 & 0,53 & 0,77 & 1,04 & 0,47 & 1,42 & 1,94 & 0,74 \\
\hline $\begin{array}{l}\text { Хронические } \\
\text { болезни нижних } \\
\text { дыхательных путей }\end{array}$ & $-0,21$ & $-0,08$ & $-0,36$ & $-0,23$ & 0,07 & $-0,39$ & $-0,14$ & $-0,12$ & $-0,16$ \\
\hline Диа & 0,01 & $-0,03$ & 0,05 & 0,01 & $-0,02$ & 0,05 & $-0,05$ & $-0,10$ & 0,03 \\
\hline Грипп и пневмония & 0,38 & 0,42 & 0,32 & 0,32 & 0,36 & 0,27 & 0,67 & 0,73 & 0,57 \\
\hline $\begin{array}{l}\text { Нефрит, } \\
\text { нефротический } \\
\text { синдром и нефроз }\end{array}$ & $-0,06$ & $-0,05$ & $-0,07$ & $-0,05$ & $-0,05$ & $-0,06$ & $-0,08$ & $-0,06$ & $-0,11$ \\
\hline Сепсис & $-0,08$ & $-0,07$ & $-0,10$ & $-0,07$ & $-0,06$ & $-0,09$ & $-0,16$ & $-0,13$ & $-0,18$ \\
\hline $\begin{array}{l}\text { Хронические } \\
\text { заболевания печени } \\
\text { и цирроз печени }\end{array}$ & 0,17 & 0,20 & 0,14 & 0,14 & 0,17 & 0,12 & 0,32 & 0,33 & 0,28 \\
\hline $\begin{array}{l}\text { Гипертензия и } \\
\text { гипертонические } \\
\text { болезни почек }\end{array}$ & 0,00 & 0,01 & $-0,01$ & 0,00 & 0,01 & $-0,01$ & 0,02 & 0,03 & 0,00 \\
\hline ричины & 1,10 & 1 , & 0 & 0,95 & 1,14 & 0 , & 1,61 & 1,59 & 1,5 \\
\hline & 6,25 & 7,2 & 4,9 & 5,85 & 6,93 & 4,50 & 6,96 & 6,99 & 6,40 \\
\hline Общее изменение $e_{0}$ & 6,26 & 7,27 & 4,94 & 5,86 & 6,91 & 4,53 & 6,96 & 6,99 & 6,40 \\
\hline
\end{tabular}

Источник: См. табл. 1 и уравнение (2).

\section{2 Таблицы смертности при условии устранения отдельных причин смерти}

Таблица 4 и рисунок 2 показывают выигрыш в продолжительности жизни от устранения той или иной причины смерти в 1970 г. и 2000 г., оцененный с использованием формулы (1). Столбец 3 в таблице 4 отражает также изменение этой величины за 1970-2000 гг. Эти значения соответствуют " $\dot{D}_{i}(0) "$ в формуле (3). Столбец 4 (красная заливка на рисунке 2 ) и столбец 5 (синяя заливка на рисунке 2) таблицы 4 соответствуют двум слагаемым правой части формулы (3). Столбец 5 - просто изменение продолжительности жизни, относимое на счет определенной причины смерти (т.е. результат нашей декомпозиции), в то время как столбец 4 показывает, как изменяется важность данной причины смерти, что определяется динамикой смертности от всех других причин в исследуемый период времени. Без учета влияния других причин столбцы 3 и 5 были бы идентичными. 


\section{Таблица 4 Декомпозиция изменений в выигрыше в ожидаемой продолжительности жизни при рождении при условии устранения определенной причины смерти в США, по полу и расовому признаку, 1970-2000 гг., лет}

\begin{tabular}{|c|c|c|c|c|c|}
\hline \multirow[t]{3}{*}{ Причины смерти } & \multicolumn{2}{|c|}{$\begin{array}{c}\text { Выигрыш в } e_{0} \text { при } \\
\text { устранении } \\
\text { причины смерти }\end{array}$} & \multirow{2}{*}{$\begin{array}{c}\text { Изменение } \\
\text { выигрыша в } e_{0} \\
\text { при устранении } \\
\text { причины } \\
\text { смерти }\end{array}$} & \multicolumn{2}{|c|}{ Уравнение (3) } \\
\hline & 1970 & 2000 & & $\begin{array}{c}\text { Первое } \\
\text { слагаемое * }\end{array}$ & $\begin{array}{c}\text { Второе } \\
\text { слагаемое }\end{array}$ \\
\hline & (1) & (2) & $\begin{array}{l}(3)=(2)-(1) \\
(3)=(4)+(5)\end{array}$ & (4) & (5) \\
\hline \multicolumn{6}{|c|}{ ВСЕ НАСЕЛЕНИЕ, ОБА ПОЛА } \\
\hline Болезни сердца & 6,20 & 3,93 & $-2,27$ & 0,60 & $-2,87$ \\
\hline $\begin{array}{l}\text { Злокачественные } \\
\text { новообразования }\end{array}$ & 2,54 & 3,27 & 0,74 & 1,00 & $-0,27$ \\
\hline $\begin{array}{l}\text { Цереброваскулярные } \\
\text { заболевания }\end{array}$ & 1,26 & 0,70 & $-0,56$ & 0,34 & $-0,90$ \\
\hline Насильственные смерти & 1,86 & 1,20 & $-0,66$ & 0,24 & $-0,90$ \\
\hline $\begin{array}{l}\text { Хронические болезни } \\
\text { нижних дыхательных путей }\end{array}$ & 0,20 & 0,55 & 0,35 & 0,15 & 0,21 \\
\hline Диабет & 0,25 & 0,34 & 0,10 & 0,11 & $-0,01$ \\
\hline Грипп и пневмония & 0,50 & 0,25 & $-0,25$ & 0,13 & $-0,38$ \\
\hline $\begin{array}{l}\text { Нефрит, нефротический } \\
\text { синдром и нефроз }\end{array}$ & 0,07 & 0,16 & 0,09 & 0,04 & 0,06 \\
\hline Сепсис & 0,03 & 0,14 & 0,11 & 0,02 & 0,08 \\
\hline $\begin{array}{l}\text { Хронические заболевания } \\
\text { печени и цирроз печени }\end{array}$ & 0,29 & 0,17 & $-0,12$ & 0,05 & $-0,17$ \\
\hline $\begin{array}{l}\text { Гипертензия и } \\
\text { гипертонические болезни } \\
\text { почек }\end{array}$ & 0,05 & 0,07 & 0,02 & 0,02 & 0,00 \\
\hline $\begin{array}{l}\text { Другие причины } \\
\text { ВСЕ НАСЕЛЕНИЕ, МУЖЧ }\end{array}$ & 3,21 & 2,99 & $-0,22$ & 0,88 & $-1,10$ \\
\hline Болезни сердца & 6,39 & 4,07 & $-2,32$ & 0,91 & $-3,22$ \\
\hline $\begin{array}{l}\text { Злокачественные } \\
\text { новообразования }\end{array}$ & 2,38 & 3,32 & 0,94 & 1,29 & $-0,35$ \\
\hline $\begin{array}{l}\text { Цереброваскулярные } \\
\text { заболевания }\end{array}$ & 0,98 & 0,57 & $-0,40$ & 0,37 & $-0,77$ \\
\hline Насильственные смерти & 2,51 & 1,66 & $-0,84$ & 0,39 & $-1,23$ \\
\hline $\begin{array}{l}\text { Хронические болезни } \\
\text { нижних дыхательных путей }\end{array}$ & 0,26 & 0,54 & 0,28 & 0,20 & 0,08 \\
\hline Диабет & 0,18 & 0,32 & 0,14 & 0,11 & 0,03 \\
\hline Грипп и пневмония & 0,50 & 0,24 & $-0,26$ & 0,16 & $-0,42$ \\
\hline $\begin{array}{l}\text { Нефрит, нефротический } \\
\text { синдром и нефроз }\end{array}$ & 0,07 & 0,16 & 0,09 & 0,05 & 0,05 \\
\hline Сепсис & 0,04 & 0,13 & 0,09 & 0,03 & 0,07 \\
\hline $\begin{array}{l}\text { Хронические заболевания } \\
\text { печени и цирроз печени }\end{array}$ & 0,33 & 0,22 & $-0,11$ & 0,09 & $-0,20$ \\
\hline $\begin{array}{l}\text { Гипертензия и } \\
\text { гипертонические болезни } \\
\text { почек }\end{array}$ & 0,05 & 0,06 & 0,01 & 0,02 & $-0,01$ \\
\hline Другие причины & 3,24 & 2,88 & $-0,35$ & 0,93 & $-1,29$ \\
\hline
\end{tabular}


ВСЕ НАСЕЛЕНИЕ, ЖЕНЩИНЫ

Болезни сердца

Злокачественные

новообразования

Цереброваскулярные

заболевания

Насильственные смерти

Хронические болезни

нижних дыхательных путей

Диабет

Грипп и пневмония

Нефрит, нефротический

синдром и нефроз

Сепсис

Хронические заболевания

печени и цирроз печени

Гипертензия и

гипертонические болезни

почек

Другие причины
БЕЛОЕ НАСЕЛЕНИЕ, ОБА ПОЛА

Болезни сердца

Злокачественные

новообразования

Цереброваскулярные

заболевания

Насильственные смерти

Хронические болезни

нижних дыхательных путей

Диабет

Грипп и пневмония

Нефрит, нефротический

синдром и нефроз

Сепсис

Хронические заболевания

печени и цирроз печени

Гипертензия и

гипертонические болезни

почек

Другие причины
$5,67 \quad 3,61$

2,61

3,17

$1,55 \quad 0,79$

0,66

1,08

0,56

$0,11 \quad 0,56$

$0,32 \quad 0,36$

$0,47 \quad 0,25$

$0,06 \quad 0,16$

$0,03 \quad 0,15$

0,23

0,11

0,05

0,07

3,08

2,97

6,22

3,85

2,56

3,27

1,19

0,66

1,71

1,17

$0,21 \quad 0,58$

$0,23 \quad 0,31$

$0,44 \quad 0,24$

0,05

0,14

0,03

0,12

0,27

0,17

0,04

0,05

2,91

2,81

0,02

$-0,10$

$-2,36$

0,72

$-0,53$

$-0,55$

0,38

0,08

$-0,19$

0,08

0,10

$-0,09$

0,02

0,84

0,32

0,22

0,15

0,10

0,13

0,03

0,02

0,05

0,00

$-0,95$ 
БЕЛОЕ НАСЕЛЕНИЕ, МУЖЧИНЫ

\begin{tabular}{|c|c|c|c|c|c|}
\hline & & & & & \\
\hline Болезни сердца & 6,49 & 4,03 & $-2,47$ & 0,86 & $-3,32$ \\
\hline $\begin{array}{l}\text { Злокачественные } \\
\text { новообразования }\end{array}$ & 2,39 & 3,29 & 0,91 & 1,27 & $-0,36$ \\
\hline $\begin{array}{l}\text { Цереброваскулярные } \\
\text { заболевания }\end{array}$ & 0,92 & 0,54 & $-0,38$ & 0,35 & $-0,73$ \\
\hline Насильственные смерти & 2,28 & 1,60 & $-0,68$ & 0,36 & $-1,04$ \\
\hline $\begin{array}{l}\text { Хронические болезни } \\
\text { нижних дыхательных путей }\end{array}$ & 0,27 & 0,56 & 0,28 & 0,21 & 0,07 \\
\hline Диабет & 0,17 & 0,30 & 0,13 & 0,10 & 0,02 \\
\hline Грипп и пневмония & 0,44 & 0,23 & $-0,21$ & 0,15 & $-0,36$ \\
\hline $\begin{array}{l}\text { Нефрит, нефротический } \\
\text { синдром и нефроз }\end{array}$ & 0,05 & 0,14 & 0,09 & 0,04 & 0,05 \\
\hline Сепсис & 0,03 & 0,12 & 0,08 & 0,03 & 0,06 \\
\hline $\begin{array}{l}\text { Хронические заболевания } \\
\text { печени и цирроз печени }\end{array}$ & 0,31 & 0,22 & $-0,08$ & 0,08 & $-0,17$ \\
\hline $\begin{array}{l}\text { Гипертензия и } \\
\text { гипертонические болезни } \\
\text { почек }\end{array}$ & 0,03 & 0,05 & 0,01 & 0,02 & $-0,01$ \\
\hline Другие причины & 2,94 & 2,69 & $-0,25$ & 0,89 & $-1,14$ \\
\hline БЕЛОЕ НАСЕЛЕНИЕ, ЖЕН & & & & & \\
\hline Болезни сердца & 5,57 & 3,48 & $-2,08$ & 0,22 & $-2,30$ \\
\hline $\begin{array}{l}\text { Злокачественные } \\
\text { новообразования }\end{array}$ & 2,64 & 3,19 & 0,55 & 0,70 & $-0,15$ \\
\hline $\begin{array}{l}\text { Цереброваскулярные } \\
\text { заболевания }\end{array}$ & 1,46 & 0,75 & $-0,71$ & 0,25 & $-0,96$ \\
\hline Насильственные смерти & 1,03 & 0,66 & $-0,37$ & 0,10 & $-0,47$ \\
\hline $\begin{array}{l}\text { Хронические болезни } \\
\text { нижних дыхательных путей }\end{array}$ & 0,10 & 0,60 & 0,49 & 0,10 & 0,39 \\
\hline Диабет & 0,29 & 0,31 & 0,03 & 0,08 & $-0,05$ \\
\hline Грипп и пневмония & 0,42 & 0,25 & $-0,17$ & 0,10 & $-0,27$ \\
\hline $\begin{array}{l}\text { Нефрит, нефротический } \\
\text { синдром и нефроз }\end{array}$ & 0,05 & 0,13 & 0,08 & 0,02 & 0,06 \\
\hline Сепсис & 0,03 & 0,13 & 0,10 & 0,02 & 0,09 \\
\hline $\begin{array}{l}\text { Хронические заболевания } \\
\text { печени и цирроз печени }\end{array}$ & 0,20 & 0,11 & $-0,09$ & 0,03 & $-0,12$ \\
\hline $\begin{array}{l}\text { Гипертензия и } \\
\text { гипертонические болезни } \\
\text { почек }\end{array}$ & 0,04 & 0,06 & 0,02 & 0,01 & 0,01 \\
\hline Другие причины & 2,79 & 2,80 & 0,01 & 0,75 & $-0,73$ \\
\hline
\end{tabular}


ЧЕРНОЕ НАСЕЛЕНИЕ, ОБА ПОЛА

\begin{tabular}{|c|c|c|c|c|c|}
\hline Болезни сердца & 6,02 & 4,42 & $-1,60$ & 0,56 & $-2,16$ \\
\hline $\begin{array}{l}\text { Злокачественные } \\
\text { новообразования }\end{array}$ & 2,42 & 3,44 & 1,03 & 1,01 & 0,02 \\
\hline $\begin{array}{l}\text { Цереброваскулярные } \\
\text { заболевания }\end{array}$ & 1,81 & 0,87 & $-0,94$ & 0,28 & $-1,21$ \\
\hline Насильственные смерти & 2,60 & 1,49 & $-1,12$ & 0,31 & $-1,42$ \\
\hline $\begin{array}{l}\text { Хронические болезни } \\
\text { нижних дыхательных путей }\end{array}$ & 0,14 & 0,36 & 0,21 & 0,08 & 0,14 \\
\hline Диабет & 0,37 & 0,58 & 0,21 & 0,16 & 0,05 \\
\hline Грипп и пневмония & 0,80 & 0,26 & $-0,55$ & 0,12 & $-0,67$ \\
\hline $\begin{array}{l}\text { Нефрит, нефротический } \\
\text { синдром и нефроз }\end{array}$ & 0,16 & 0,32 & 0,15 & 0,07 & 0,08 \\
\hline Сепсис & 0,06 & 0,27 & 0,21 & 0,05 & 0,16 \\
\hline $\begin{array}{l}\text { Хронические заболевания } \\
\text { печени и цирроз печени }\end{array}$ & 0,41 & 0,15 & $-0,25$ & 0,07 & $-0,32$ \\
\hline $\begin{array}{l}\text { Гипертензия и } \\
\text { гипертонические болезни } \\
\text { почек }\end{array}$ & 0,14 & 0,17 & 0,03 & 0,05 & $-0,02$ \\
\hline $\begin{array}{l}\text { Другие причины } \\
\text { ЧЕРНОЕ НАСЕЛЕНИЕ, МУ }\end{array}$ & $\begin{array}{l}4,84 \\
b I\end{array}$ & 4,07 & $-0,77$ & 0,84 & $-1,61$ \\
\hline Болезни сердца & 5,40 & 4,17 & $-1,23$ & 0,67 & $-1,909$ \\
\hline $\begin{array}{l}\text { Злокачественные } \\
\text { новообразования }\end{array}$ & 2,33 & 3,59 & 1,25 & 1,19 & 0,06 \\
\hline $\begin{array}{l}\text { Цереброваскулярные } \\
\text { заболевания }\end{array}$ & 1,40 & 0,73 & $-0,66$ & 0,28 & $-0,94$ \\
\hline Насильственные смерти & 3,72 & 2,20 & $-1,51$ & 0,43 & $-1,94$ \\
\hline $\begin{array}{l}\text { Хронические болезни } \\
\text { нижних дыхательных путей }\end{array}$ & 0,17 & 0,38 & 0,21 & 0,10 & 0,12 \\
\hline Диабет & 0,23 & 0,45 & 0,22 & 0,12 & 0,10 \\
\hline Грипп и пневмония & 0,85 & 0,26 & $-0,59$ & 0,14 & $-0,73$ \\
\hline $\begin{array}{l}\text { Нефрит, нефротический } \\
\text { синдром и нефроз }\end{array}$ & 0,15 & 0,28 & 0,13 & 0,07 & 0,06 \\
\hline Сепсис & 0,06 & 0,24 & 0,18 & 0,04 & 0,13 \\
\hline $\begin{array}{l}\text { Хронические заболевания } \\
\text { печени и цирроз печени }\end{array}$ & 0,43 & 0,18 & $-0,25$ & 0,08 & $-0,33$ \\
\hline $\begin{array}{l}\text { Гипертензия и } \\
\text { гипертонические болезни } \\
\text { почек }\end{array}$ & 0,13 & 0,14 & 0,01 & 0,05 & $-0,03$ \\
\hline Другие причины & 4,82 & 4,08 & $-0,74$ & 0,86 & $-1,59$ \\
\hline
\end{tabular}




\section{ЧЕРНОЕ НАСЕЛЕНИЕ, ЖЕНЩИНЫ}

\begin{tabular}{|c|c|c|c|c|c|}
\hline Болезни сердца & 6,49 & 4,45 & $-2,04$ & 0,23 & $-2,27$ \\
\hline $\begin{array}{l}\text { Злокачественные } \\
\text { новообразования }\end{array}$ & 2,40 & 3,22 & 0,82 & 0,77 & 0,04 \\
\hline $\begin{array}{l}\text { Цереброваскулярные } \\
\text { заболевания }\end{array}$ & 2,26 & 0,98 & $-1,28$ & 0,20 & $-1,48$ \\
\hline Насильственные смерти & 1,29 & 0,69 & $-0,60$ & 0,14 & $-0,74$ \\
\hline $\begin{array}{l}\text { Хронические болезни } \\
\text { нижних дыхательных путей }\end{array}$ & 0,10 & 0,31 & 0,21 & 0,05 & 0,16 \\
\hline Диабет & 0,55 & 0,68 & 0,14 & 0,17 & $-0,03$ \\
\hline Грипп и пневмония & 0,72 & 0,24 & $-0,48$ & 0,09 & $-0,57$ \\
\hline $\begin{array}{l}\text { Нефрит, нефротический } \\
\text { синдром и нефроз }\end{array}$ & 0,17 & 0,35 & 0,17 & 0,07 & 0,11 \\
\hline Сепсис & 0,07 & 0,30 & 0,23 & 0,04 & 0,18 \\
\hline $\begin{array}{l}\text { Хронические заболевания } \\
\text { печени и цирроз печени }\end{array}$ & 0,35 & 0,12 & $-0,24$ & 0,05 & $-0,28$ \\
\hline $\begin{array}{l}\text { Гипертензия и } \\
\text { гипертонические болезни } \\
\text { почек }\end{array}$ & 0,15 & 0,19 & 0,04 & 0,04 & 0,00 \\
\hline Другие причины & 4,71 & 3,91 & $-0,80$ & 0,72 & $-1,51$ \\
\hline
\end{tabular}

Примечания: * - эффект влияния изменений смертности от других причин смерти на число потерянных лет жизни от данной причинь; ** - эффект влияния изменения смертности от данной причины на число потерянных лет жизни.

Источник: См. табл.1 и уравнение (3).

Таблица 4 указывает на меняющееся значение рака и болезней сердца как причин смерти. Именно за счет устранения этих двух групп причин можно было бы добиться наибольшего увеличения ожидаемой продолжительности жизни при рождении (столбцы 1 и 2) как в 1970 г., так и в 2000 г. Но относительное значение этих двух причин драматически изменилось. В 1970 г. потери продолжительности жизни от болезней сердца для всех групп населения были примерно в 2,4 раза больше потерь продолжительности жизни от злокачественных новообразований. В 2000 г. это соотношение составило 1,2 раза.

Несмотря на то, что в 1970-2000 гг. смертность от злокачественных новообразований медленно снижалась, в 2000 г. число лет жизни, потерянных из-за рака, было большее, чем в 1970 г. Среднее число лет жизни, потерянных из-за злокачественных новообразований, для всего населения выросло на 0,74 года, что больше по сравнению с любой другой причиной. Это значение, показанное в столбце 3 , представляет собой сумму вклада в $+1,00$ года в результате снижения смертности от «других причин» (столбец 4), частично компенсированного на величину в $-0,27$ года в результате снижения смертности от самих злокачественных новообразований. Другими словами, основная причина того, что число потерянных лет жизни от рака в 2000 году больше, чем оно было в 1970, - быстрое снижение смертности от других причин, в особенности от болезней сердца. 


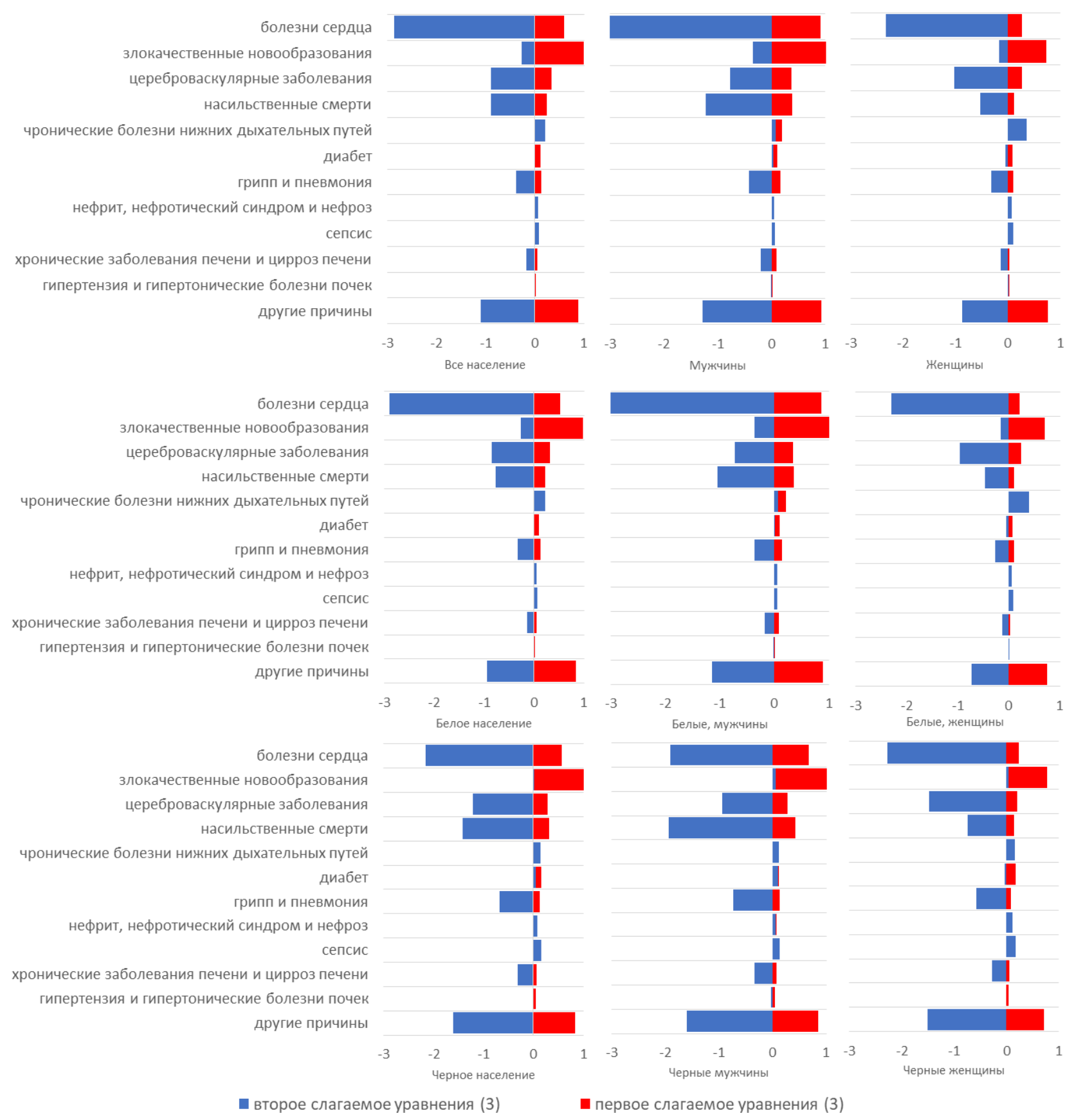

Рисунок 1 Декомпозиция изменений в выигрыше в ожидаемой продолжительности жизни при рождении при условии устранения определенной причины смерти в США, по полу и расовому признаку, 1970-2000 гг., лет

Примечания: * - эффект изменения смертности от других причин смерти на число потерянных лет жизни от данной причины; ** - эффект изменения смертности от данной причины на число потерянных лет жизни.

Источник: См. табл. 4.

Если бы уровень смертности от рака не изменялся в 1970-2000 гг., то число лет жизни, потерянных из-за рака в 2000 году составило бы 1,00 года. Из-за снижения смертности от других причин смерти, кроме рака, те, кто умер от рака в 2000 г., в среднем прожили бы дольше, чем те, кто умер от рака в 1970 г. В результате, рак вызвал большие 
потери в продолжительности жизни в 2000 г., чем в 1970 г., несмотря на то, что уровень смертности от рака снижался на протяжении всего периода. Этот результат подчеркивает важность взаимодействия причин смерти в оценке продолжительности жизни. Ключевое взаимодействие явно в наглядном виде показано в уравнении (3).

В таком кажущемся необычным результате, касающемся рака, нет ничего неожиданного. В самом деле, значения в столбце 4 - положительные для всех причины смерти и всех групп населения, приведенных в таблице 4. Снижение смертности от одних причин, при прочих равных условиях, неизбежно ведет к увеличению потерянных лет жизни от каких-либо иных причины смерти. В случае болезней сердца, снижение смертности от других причин увеличило количество потерянных лет жизни от болезней сердца на 0,60 года между 1970 и 2000 гг. Но само по себе снижение смертности от болезней сердца было достаточно значительным (2,87 года), чтобы превзойти этот эффект и сократить количество потерянных лет жизни от болезней сердца на 2,27 года.

\section{6. ЗАКЛЮЧЕНИЕ}

Таблицы смертности при условии устранения той или иной причины смерти ясно указывают на значение для общественного здоровья конкретных заболеваний или травм. Декомпозиция изменений продолжительности жизни оценивает вклад конкретных причин смерти в этих изменения. В нашей статье мы показываем, что оба типа анализа тесно связаны друг с другом. В частности, показано, что изменение числа лет жизни, потерянных из-за той или иной причины смерти, равно сумме двух компонент - вклада данной причины в изменения ожидаемой продолжительности жизни (метод декомпозиции) и компоненты, отражающей динамику смертности от остальных причин смерти. Таким образом, значение определенного заболевания для общественного здоровья - результат «борьбы» между причинами смерти. Если смертность от одной причины снижается быстрее, чем от других причин, как показано в уравнении (3), то данная причина «отнимет» меньшее количество лет в продолжительности жизни к концу исследуемого периода. Болезни сердца выиграли эту борьбу за рассматриваемый период, в то время как снижение смертности от рака было недостаточным для того, чтобы к концу периода число потерянных лет жизни от рака стало меньше, чем в начале периода.

Простота формул дополняется простотой расчетов. Для применения метода декомпозиции, описанного уравнением (2), необходимо построить таблицы смертности при условии устранения причин смерти. Стало быть, построение таких таблиц - важный промежуточный элемент рассматриваемого здесь метода декомпозиции. В итоге, полные результаты расчетов, описанные уравнениями (2) и (3) и представленные в таблице 4, могут быть получены, когда выполнена декомпозиция. Они могут быть также получены при построении таблиц смертности при условии устранения причин смерти для двух временных точек. 


\section{ЛИТЕРАТУРА}

Андреев Е.М. (1982). Метод компонент в анализе продолжительности жизни // Вестник статистики. 9: 42-47.

Andreev E, V.M. Shkolnikov, A.Z. Begun (2002). Algorithm for decomposition of differences between aggregate demographic measures and its application to life expectancies, healthy life expectancies, parity progression ratios and total fertility rates // Demographic Research.7(14):499R.

Arias E. (2002). United States Life Tables, 2000. Hyattsville, MD: National Center for Health Statistics.

Arriaga E. (1982). A note on the use of temporary life expectancies for analyzing changes and differentials of mortality / Mortality in South and East Asia: A Review of Changing Trends and Patterns, Manila 1980, WHO/ESCAP. Geneva: World Health Organization: 559-562.

- (1984). Measuring and explaining the change in life expectancies // Demography. 21(1):83-96.

(1989). Changing trends in mortality decline during the last decade / L. Ruzicka, W. Guillaume, P. Kane, eds. Differential Mortality: Methodological Issues and Biosocial Factors. Oxford, England: Clarendon, Press. International Studies in Demography: 105-129..

Beltrán-Sánchez H., S.H. Preston (2007). A new method for attributing changes in life expectancy to various causes of death, with application to the United States // PSC Working Paper Series PSC 07-01. URL:

http://repository.upenn.edu/cgi/viewcontent.cgi?article=1004\&context=psc_working_ papers.

Brownlee J. (1919). Notes on the biology of a life-table // Journal of Royal Statistical Society. 82(1):34-77.

Centers for Disease Control (2001). Comparability across ICD revisions for selected causes. URL: http://www.cdc.gov/nchs/data/statab/comp2.pdf.

Centers for Disease Control (2005). 10 leading causes of death, United States 2000, all races, both sexes. URL: http://webappa.cdc.gov/sasweb/ncipc/leadcaus10.html.

Chiang L. (1968). Introduction to Stochastic Processes in Biostatistics. New York: John Wiley and Sons.

Cutler D. (2004). Your Money or Your Life. Oxford, England: Oxford University Press.

Ergin A., P. Muntner, R. Sherwin, J. He (2004). Secular trends in cardiovascular disease mortality, incidence, and case fatality rates in adults in the United States // American Journal of Medicine, 117:219-227.

Fisher A., E. Vigfusson, C. Dickson (1922). An Elementary Treatise on Frequency Curves and Their Application in the Analysis of Death Curves and Life Tables. New York: The Macmillan Co.

Ford E.S., U. Ajani, J. Croft, J. Critchley, D. Labarthe, T. Kottke, W.Gile, S. Capewell (2007). Explaining the decrease in U. S. deaths from coronary disease, 1980-2000 // New England Journal of Medicine. 356(23):2388-2398.

Greville T. (1948). Mortality tables analyzed by cause of death // The Record. 37(76):283- 294. 
Inter-university Consortium for Political and Social Research (2004). Multiple Cause of Death, 1968-1973. Ann Arbor, Mich.: Inter-university Consortium for Political and Social Research [distributor]. URL:http://webapp.icpsr.umich.edu/cocoon/ICPSRSTUDY/03905.xml.

(2007). Multiple Cause of Death Public Use Files, 2000-2002. Ann Arbor, Mich.: Inter-university Consortium for Political and Social Research [distributor]. URL: http://webapp.icpsr.umich.edu/cocoon/ICPSR-STUDY/04640.xml.

Jordan C. (1952). Life Contingencies. Chicago, Ill.: Society of Actuaries.

National Center for Health Statistics (1974). Vital Statistics of the United States, 1970: Life Tables. Vol. 2, Sec.5. Washington, DC: U.S. Government Printing Office.

Pearl R. (1922). The Biology of Death; Beinga Series of Lectures Delivered at the Lowell Institute in Boston in December 1920. Philadelphia; London: J.B. Lippincott Company.

Pollard J. (1982). The expectation of life and its relationship to mortality // Journal of Institute of Actuaries. 109 (Part II, No 442):225-240.

(1988). On the decomposition of changes in expectation of life and differentials in life expectancy // Demography. 25(2):265-276.

Pressat R. (1985). Contribution des écarts de mortalité par âge à la différence des vies moyennes // Population. 4-5:765-770.

Preston S., I. Elo, A. Foster, H. Fu. (1998). Reconstructing the size of the African American population by age and sex, 1930-1990 // Demography. 35(1):1-21.

Preston S., P. Heuveline, M. Guillot (2001). Demography. Measuring and Modeling Population Processes. Malden, Massachusetts: Blackwell Publishers Inc., 2002 edition.

Preston S., N. Keyfitz, R. Schoen (1972). Causes of Death: Life Tables for National Populations. New York and London: Seminar Press.

Siegel J. (1974). Estimates of coverage of population by sex, race, and age in 1970 census // Demography. 11(1):1-23.

Siegel J., J. Passel (1976). New estimates of number of centenarians in United States // Journal of the American Statistical Association. 71(355):559-566.

Spiegelman, M. (1968). Introduction to Demography. Cambridge, Mass.: Harvard University Press.

United States (1972). 1970 census of population and housing: General Population Characteristics. Washington, DC.: U.S. Dept. of Commerce, Social and Economic Statistics Administration, Bureau of the Census.

United States Department of Health, Education, and Welfare (1968). United States Life Tables by Causes of Death: 1959-1961. (Life Tables: 1959-61, Volume 1, No. 6). Washington, D.C.: U.S. Government Printing Office.

U.S. Bureau of the Census (2005). Monthly postcensal resident population, by single year of age, sex, race, and Hispanic origin, 2000-2005. URL: http://www.census.gov/popest/national/asrh/2003_nat_res.html.

Vaupel J., V. Canudas-Romo (2002). Decomposing demographic change into direct vs. compositional components // Demographic Research. 7(1):1-14.

(2003). Decomposing change in life expectancy: a bouquet of formulas in honor of Nathan Keyfitz's 90th birthday // Demography. 40(2): 201- 216 


\section{ПРИЛОЖЕНИЕ 1. СВЯЗЬ МЕЖДУ НАШИМ ПОДХОДОМ И ПОДХОДОМ ПОЛЛАРДА}

Как сказано выше, уравнение (2) может быть выведено из уравнения (36) Вопеля и КанудасРомо (2003:209). Они показали, что их формула эквивалентна уравнению (38) Полларда [Vaupel, Canudas-Romo 2003: 209]. Таким образом, и наше уравнение (2) тоже эквивалентно уравнению (38) Полларда, использованному в работе [Vaupel, Canudas-Romo 2003].

Применительно к данным по США, различия в оценках вклада причин смерти, выполненных нашим методом, а также методами Полларда и Арриаги, показаны в таблице П1 Приложения. Фактически между нашими оценками и оценками Полларда никакой разницы нет; самое большое расхождение составляет четыре сотых года. Если не считать болезни сердца, разница между оценками методом Арриаги и нашим также весьма незначительна. Для всего населения вклад болезней сердца, по нашей оценке, примерно на 0,1 года выше, чем в расчетах Арриаги. Таков же порядок различий для всего мужского населения, и немного ниже для женщин и всего белого населения. Для всего черного населения, черных мужчин и черных женщин наши оценки, соответственно, примерно на 6\%, 6,9\% и 5,5\% выше, чем по формуле Арриаги (данные не отображены).

Таблица П1. Разница в оценках вклада разных причин смерти в изменения ожидаемой продолжительности жизни при рождении, выполненных предлагаемым методом и методами Полларда и Арриаги, США, 1970-2000 (наши оценки минус оценки Полларда и Арриаги соответственно, лет)

\begin{tabular}{|c|c|c|c|c|c|c|c|c|c|c|}
\hline \multirow[t]{2}{*}{ Причины смерти } & \multicolumn{2}{|c|}{$\begin{array}{c}\text { Все } \\
\text { население }\end{array}$} & \multicolumn{2}{|c|}{ Мужчины } & \multicolumn{2}{|c|}{ Женщины } & \multicolumn{2}{|c|}{$\begin{array}{c}\text { Белое } \\
\text { население, } \\
\text { оба пола }\end{array}$} & \multicolumn{2}{|c|}{$\begin{array}{c}\text { Черное } \\
\text { население, } \\
\text { оба пола }\end{array}$} \\
\hline & $\begin{array}{c}\text { Аp- } \\
\text { риага }\end{array}$ & $\begin{array}{l}\text { Пол- } \\
\text { лард }\end{array}$ & $\begin{array}{c}\text { Ap- } \\
\text { риага }\end{array}$ & $\begin{array}{l}\text { Пол- } \\
\text { лард }\end{array}$ & $\begin{array}{c}\text { Ap- } \\
\text { риага }\end{array}$ & $\begin{array}{l}\text { Пол- } \\
\text { лард }\end{array}$ & $\begin{array}{c}\text { Аp- } \\
\text { риага }\end{array}$ & $\begin{array}{l}\text { Пол- } \\
\text { лард }\end{array}$ & $\begin{array}{c}\text { Ap- } \\
\text { риага }\end{array}$ & $\begin{array}{l}\text { Пол- } \\
\text { лард }\end{array}$ \\
\hline Болезни сердца & 0,10 & 0,00 & 0,11 & $-0,01$ & 0,07 & 0,00 & 0,09 & 0,00 & 0,13 & 0,02 \\
\hline $\begin{array}{l}\text { Злокачественные } \\
\text { новообразования }\end{array}$ & 0,07 & 0,01 & 0,08 & 0,01 & 0,06 & 0,01 & 0,07 & 0,01 & 0,09 & 0,01 \\
\hline $\begin{array}{l}\text { Цереброваскулярные } \\
\text { заболевания }\end{array}$ & $-0,03$ & 0,00 & $-0,04$ & 0,00 & $-0,02$ & 0,00 & $-0,03$ & 0,00 & $-0,05$ & 0,00 \\
\hline Насильственные смерти & $-0,03$ & $-0,01$ & $-0,03$ & $-0,01$ & $-0,02$ & $-0,01$ & $-0,03$ & $-0,01$ & $-0,02$ & 0,00 \\
\hline $\begin{array}{l}\text { Хронические болезни } \\
\text { нижних дыхательных } \\
\text { путей }\end{array}$ & $-0,01$ & 0,01 & $-0,03$ & 0,01 & 0,00 & 0,00 & 0,00 & 0,01 & $-0,02$ & 0,00 \\
\hline Диабет & 0,00 & 0,00 & $-0,01$ & 0,00 & 0,0 & 0,0 & 0,00 & 0,00 & $-0,02$ & 0,00 \\
\hline Грипп и пневмония & 0,00 & 0,01 & 0,00 & 0,00 & 0,00 & 0,01 & 0,00 & 0,01 & 0,00 & 0,01 \\
\hline $\begin{array}{l}\text { Нефрит, нефротический } \\
\text { синдром и нефроз }\end{array}$ & $-0,01$ & $-0,01$ & -( & -( & -( & 0,00 & 0,00 & 0,00 & $-0,02$ & 0,00 \\
\hline Сепсис & $-0,01$ & 0,00 & $-0,01$ & $-0,01$ & $-0,01$ & 0,00 & 0,00 & 0,00 & $-0,02$ & 0,00 \\
\hline $\begin{array}{l}\text { Хронические } \\
\text { заболевания печени и } \\
\text { цирроз печени }\end{array}$ & 0,00 & 0,00 & 0,00 & 0,01 & 0,00 & 0,00 & 0,00 & 0,00 & 0,00 & 0,00 \\
\hline $\begin{array}{l}\text { Гипертензия и } \\
\text { гипертонические } \\
\text { заболевания почек }\end{array}$ & 0,00 & 0,00 & 0,00 & 0,00 & $-0,01$ & 0,00 & 0,00 & 0,00 & $-0,01$ & 0,00 \\
\hline Другие причины & $-0,10$ & $-0,03$ & $-0,07$ & $-0,01$ & $-0,10$ & $-0,04$ & $-0,09$ & $-0,03$ & $-0,07$ & 0,00 \\
\hline
\end{tabular}

Примечание: Примененная формула Арриаги выведена из уравнения в его работе [Arriaga 1989:125], где сводные таблицы смертности закрываются с помощью метода, описанного в приложении 2. Примененный подход Полларда взят из его работь [Pollard 1982:229]. Источник: см. таб.1 и 3. 


\section{ПРИЛОЖЕНИЕ 2. ПРИМЕНЕНИЕ УРАВНЕНИЙ (2) И (3) ДЛЯ ДИСКРЕТНЫХ ВРЕМЕННЫХ ИНТЕРВАЛОВ}

Для дискретных временных интервалов уравнение (2) можно записать в следующем виде [Beltrán-Sánchez, Preston 2007: Приложение 1]:

$$
e^{*}(0)-e(0) \cong \sum_{i=1}^{n} \int_{0}^{\infty}\left(p_{i}^{*}-p_{i}\right)\left(\frac{p_{-i}+p_{-i}^{*}}{2}\right) d a
$$

Чтобы привести уравнение (3) к виду для дискретного времени, вернемся к формуле (1). Прирост ожидаемой продолжительности жизни при рождении для дискретных временных интервалов при устранении причины смерти $i$ вычисляется следующим образом:

$$
D_{i}(0)=\int_{0}^{\infty} p_{-i}(a) d a-\int_{0}^{\infty} p_{i}(a) p_{-i}(a) d a,
$$

и

$$
D_{i}^{*}(0)=\int_{0}^{\infty} p_{-i}^{*}(a) d a-\int_{0}^{\infty} p_{i}^{*}(a) p_{-p}^{*}(a) d a .
$$

Для простоты пусть $p_{i}(a)=p_{i}$ и $p^{*}{ }_{i}(a)=p^{*}{ }_{i}$. Тогда разница $D^{*}{ }_{i}-D_{i}(0)$ вычисляется следующим образом:

$$
\begin{aligned}
& D_{i}^{*}(0)-D_{i}(0)=\int_{0}^{\infty} p_{-i}^{*} d a-\int_{0}^{\infty} p_{i}^{*} p_{-i}^{*} d a-\left(\int_{0}^{\infty} p_{-i} d a-\int_{0}^{\infty} p_{i} p_{-i} d a\right. \\
& D_{i}^{*}(0)-D_{i}(0)=\int_{0}^{\infty}\left(p_{-i}^{*}-p_{-i}\right) d a-\left(\int_{0}^{\infty} p_{i}^{*} p_{-i}^{*} d a-\int_{0}^{\infty} p_{i} p_{-i} d a\right)
\end{aligned}
$$

Выражение в скобках из уравнения (П2) может быть записано как:

$$
\begin{aligned}
& \int_{0}^{\infty} p_{i}^{*} p_{-p}^{*} d a-\int_{0}^{\infty} p_{i} p_{-i} d a \\
& =\int_{0}^{\infty}\left(p_{i}^{*}-p_{i}\right)\left(\frac{p_{-i}+p_{-i}^{*}}{2}\right) d a+\int_{0}^{\infty}\left(p_{-i}^{*}-p_{-i}\right)\left(\frac{p_{i}+p_{i}^{*}}{2}\right) d a .
\end{aligned}
$$

Тогда,

$$
\begin{aligned}
& D_{i}^{*}(0)-D_{i}(0) \\
& =\int_{0}^{\infty}\left(p_{-i}^{*}-p_{i}\right) d a-\int_{0}^{\infty}\left(p_{i}^{*}-p_{i}\right)\left(\frac{p_{i}+p_{-i}^{*}}{2}\right) d a-\int_{0}^{\infty}\left(p_{-i}^{*}-p_{i}\right)\left(\frac{p_{i}+p_{i}^{*}}{2}\right) d a
\end{aligned}
$$




$$
\begin{aligned}
& =\int_{0}^{\infty}\left(p_{-i}^{*}-p_{i}\right)\left(1-\frac{p_{i}+p_{i}^{*}}{2}\right) d a-\int_{0}^{\infty}\left(p_{i}^{*}-p_{i}\right)\left(\frac{p_{-i}+p_{-i}^{*}}{2}\right) d a \\
& =\int_{0}^{\infty}\left(p_{-i}^{*}-p_{i}\right)\left(\frac{q_{i}+q_{i}^{*}}{2}\right) d a-\int_{0}^{\infty}\left(p_{i}^{*}-p_{i}\right) .(\Pi 3)
\end{aligned}
$$

Таким образом, для дискретных временных интервалов уравнение (3) может быть рассчитано с помощью уравнения (П3).

\section{ПРИЛОЖЕНИЕ З. ПРИМЕНЕНИЕ УРАВНЕНИЙ (2) И (3) ДЛЯ ДИСКРЕТНЫХ ВОЗРАСТНЫХ ИНТЕРВАЛОВ}

Как показано в приложении 2, для дискретных временных интервалов уравнения (2) и (3) представлены следующим образом:

$$
e^{*}(0)-e(0) \cong \sum_{i=1}^{n} \int_{0}^{\infty}\left(p_{i}^{*}-p_{i}\right)\left(\frac{p_{-i}+p_{-i}^{*}}{2}\right) d a
$$

И

$$
D_{i}^{*}(0)-D_{i}(0)=\int_{0}^{\infty}\left(p_{-i}^{*}-p_{-i}\right)\left(\frac{q_{i}+q_{i}^{*}}{2}\right) d a-\int_{0}^{\infty}\left(p_{i}^{*}-p_{i}\right)\left(\frac{p_{-i}+p_{-i}^{*}}{2}\right) d a
$$

где $q_{i}=1-p_{i}$ и $q^{*}{ }_{i}=1-p^{*}{ }_{i}$.

Вышеприведенные формулы для дискретных возрастных интервалов эквивалентны выражениям:

$$
e^{*}(0)-e(0)=\sum_{i=1}^{n} \sum_{x=0,5}^{\omega}\left({ }_{n} L_{x, i}^{*}-{ }_{n} L_{x, i}\right)\left(\frac{{ }_{n} L_{x,-i}^{*}+{ }_{n} L_{x,-i}}{2_{n}}\right)
$$

И

$$
\begin{aligned}
D_{i}^{*}(0)-D_{i}(0) & =\sum_{x=0,5}^{\omega}\left({ }_{n} L_{x,-i}^{*}-{ }_{n} L_{x,-i}\right)\left(1-\frac{{ }_{n} L_{x, i}+{ }_{n} L_{x, i}^{*}}{2_{n}}\right) \\
& -\sum_{x=0,5}^{\omega}\left({ }_{n} L_{x, i}^{*}-{ }_{n} L_{x, i}\right)\left(\frac{{ }_{n} L_{x,-i}+{ }_{n} L_{x,-i}^{*}}{2_{n}}\right) .
\end{aligned}
$$

при корне таблиц смертности, равном $1\left(l_{0}=1\right)$, где ${ }_{n} L_{x, i},{ }_{n} L_{x,-i, n} L_{x, I, n}^{*} L_{x,-i}^{*}$ означают число человеко-лет, прожитых в возрасте от $x$ до $x+n$ в моменты 1 и 2 в таблицах смертности для причин смерти $i$ и $-i$ соответственно.

Для того, чтобы вычислить ${ }_{n} L_{x, i}$, допустим, что сила убывания функции для причины $i$ пропорциональна силе убывания функции для всех остальных причин вместе взятых на 
возрастном интервале от $x$ до $x+n$ [Preston et al. 2001: 82]. Вычисление ${ }_{n} L_{x,-i}$ также требует расчета значения $n a_{x, i}$, которое представляет среднее число человеко-лет, прожитых в возрастном интервале от $x$ до $x+n$ умершими от причины $i$ в данном интервале. Эти значения получены сглаживанием с помощью уравнения 4.8 для возрастов $0,1,5$ и 95 лет и уравнения 4.6 для возрастов от 10 до 90 лет из работы [Preston et al. 2001:82-84].

Вычислив ${ }_{n} L_{x, i}$, рассчитываем затем число прожитых человеко-лет в таблицах смертности при устранении каждой причины смерти:

$$
{ }_{n} L_{x,-i}=\left(\frac{{ }_{n} L_{x}}{{ }_{n} L_{x, i}}\right) \cdot n,
$$

где ${ }_{n} L_{x}$ - число человеко-лет, прожитых в возрасте от $x$ до $x+n$ в исходной таблице смертности от всех причин смерти на каждом временном интервале. Исходные таблицы смертности для 1970 и 2000 гг. строятся по методологии [Preston et al. 2001: гл.3], включая применение сглаживания для вычисления ${ }_{n} a_{x, i}$ как было описано выше.

Уравнение (П4) сохраняет мультипликативность, в силу чего произведение вероятностей дожития до определенного возраста во взаимосвязанных таблице единственного выбытия по причине $i$, и таблице смертности при условии устранения причины $i$ равно вероятности дожития до данного возраста, вычисленной для всех причин смерти.

Мы также хотели сохранить мультипликативное свойство когда объединяются взаимосвязанные таблицы единственного выбытия для каждой причины смерти. Нам удалось этого добиться с помощью формулы, по которой вычисляется число прожитых человеко-лет для остаточной категории, включающей все оставшиеся причины смерти, не рассмотренные по отдельности. В частности, число человеко-лет для оставшихся причин смерти (оставшаяся причина $k$ ) вычисляется следующим образом ${ }^{7}$

$$
{ }_{n} L_{x, k}=\left(\frac{n L_{x}}{\prod_{m=1}^{k-1} n_{x, m}}\right) \cdot n^{(k-1)} \text { для } \mathrm{t}=1,2 .
$$

Для верхнего открытого интервала, который в данном исследовании начинается со 100 лет, предполагается что уровень смертности остается неизменным, а также нет никаких человеко-лет, проживаемых в возрасте старше 110 лет. В данном случае, число человеколет для исходной таблицы и связанной с нею таблицы единственного выбытия вычисляется следующим образом:

\footnotetext{
7 Существует два варианта вычисления числа прожитых человеко-лет (ЧЧЛ) для сводных таблиц и для таблицы смертности при условии устранения причины смерти. С одной стороны, мы может моделировать каждую причину смерти (включая оставшиеся) как связанную таблицу и вычислить ЧЧЛ для таблицы смертности при условии устранения причины смерти как отношение, используя уравнение (П4). С другой стороны, мы можем моделировать первые $n-1$ причин смерти как связанную таблицу и вычислить ЧЧЛ для оставшихся причин смерти по уравнению (П4), а затем вычислить ЧЧЛ для таблицы смертности при условии устранения причины смерти по уравнению (П5). В полученных результатах будет небольшая разница, но мы предпочитаем использовать второй вариант, т.к. он позволяет дать более точные оценки условий взаимодействия.
} 


$$
{ }_{+} L_{100}=l_{100} \cdot \int_{100}^{110} e^{-M(a-100)} d a+l_{100} \cdot\left(-\frac{1}{M}\left(e^{-10 M}-1\right)=l_{100} \cdot \frac{1-e^{-10 M}}{M}\right.
$$

и

$$
{ }_{+} L_{100}^{i}=L_{100}^{i} \cdot \frac{1-e^{-10 M^{i}}}{M^{i}}
$$

соответственно, где $M$ и $M^{i}$ - коэффициенты смертности в возрастах старше 100 лет от всех причин смерти и от причины $i$. 


\title{
AN INTEGRATED APPROACH TO CAUSE-OF-DEATH
}

\section{ANALYSIS: CAUSE-DELETED LIFE TABLES AND DECOMPOSITIONS OF LIFE EXPECTANCY}

\author{
Hiram Beltran-Sanchez, SAmuel Preston, Vladimir Canudas-Romo
}

\begin{abstract}
This article integrates two methods that analyze the implications of various causes of death for life expectancy. One of the methods attributes changes in life expectancy to various causes of death; the other method examines the effect of removing deaths from a particular cause on life expectancy. This integration is accomplished by new formulas that make clearer the interactions among causes of death in determining life expectancy. We apply our approach to changes in life expectancy in the United States between 1970 and 2000. We demonstrate, and explain analytically, the paradox that cancer is responsible for more years of life lost in 2000 than in 1970 despite the fact that declines in cancer mortality contributed to advances in life expectancy between 1970 and 2000.
\end{abstract}

Key words: life tables, decomposition of life expectancy, causes of death, cause-deleted life tables, United States.

Hiram Beltran-Sanchez, (beltrans@ucla.edu), UCla Fielding School of Public Health, USA.

Samuel Preston, University of Pennsylvania, USA.

Vladimir Canudas-Romo, Australian National University, AuStralia.

REPRINTED WITH THE PERMISSION OF THE DEMOGRAPHIC RESEARCH FROM: BELTRAN-SANCHEZ H., S.H. PRESTON, V. CANUDAS-ROMO (2008). AN INTEGRATED APPROACH TO CAUSE-OF-DEATH ANALYSIS: CAUSE-DELETED LIFE TABLES AND DECOMPOSITIONS OF LIFE EXPECTANCY // DEMOGRAPHIC RESEARCH. 19 (35): 1323-135O.

DOI: HTTPS://DX.DOI.ORG/10.4054/DEMRES.2008.19.35

\section{REFERENCES}

Andreev E.M. (1982). Metod komponent v analize prodolzhitel'nosti zhizni [Method of components in the analysis of life expectancy] // Vestnik statistiki [Bulletin of Statistics]. 9: $42-47$

Andreev E, V.M. Shkolnikov, A.Z. Begun (2002). Algorithm for decomposition of differences between aggregate demographic measures and its application to life expectancies, healthy life expectancies, parity progression ratios and total fertility rates // Demographic Research.7(14):499R.

Arias E. (2002). United States Life Tables, 2000. Hyattsville, MD: National Center for Health Statistics.

Arriaga E. (1982). A note on the use of temporary life expectancies for analyzing changes and differentials of mortality / Mortality in South and East Asia: A Review of Changing Trends and Patterns, Manila 1980, WHO/ESCAP. Geneva: World Health Organization: 559-562. (1984). Measuring and explaining the change in life expectancies // Demography. 21(1):83-96.

(1989). Changing trends in mortality decline during the last decade / L. Ruzicka, W. Guillaume, P. Kane, eds. Differential Mortality: Methodological Issues and Biosocial Factors. Oxford, England: Clarendon, Press. International Studies in Demography: 105-129.. 
Beltrán-Sánchez H., S.H. Preston (2007). A new method for attributing changes in life expectancy to various causes of death, with application to the United States // PSC Working Paper Series PSC 07-01. URL:

http://repository.upenn.edu/cgi/viewcontent.cgi?article=1004\&context=psc_working_ papers.

Brownlee J. (1919). Notes on the biology of a life-table // Journal of Royal Statistical Society. 82(1):34-77.

Centers for Disease Control (2001). Comparability across ICD revisions for selected causes. URL: http://www.cdc.gov/nchs/data/statab/comp2.pdf.

Centers for Disease Control (2005). 10 leading causes of death, United States 2000, all races, both sexes. URL: http://webappa.cdc.gov/sasweb/ncipc/leadcaus 10.html.

Chiang L. (1968). Introduction to Stochastic Processes in Biostatistics. New York: John Wiley and Sons.

Cutler D. (2004). Your Money or Your Life. Oxford, England: Oxford University Press.

Ergin A., P. Muntner, R. Sherwin, J. He (2004). Secular trends in cardiovascular disease mortality, incidence, and case fatality rates in adults in the United States // American Journal of Medicine, 117:219-227.

Fisher A., E. Vigfusson, C. Dickson (1922). An Elementary Treatise on Frequency Curves and Their Application in the Analysis of Death Curves and Life Tables. New York: The Macmillan Co.

Ford E.S., U. Ajani, J. Croft, J. Critchley, D. Labarthe, T. Kottke, W.Gile, S. Capewell (2007). Explaining the decrease in U. S. deaths from coronary disease, 1980-2000 // New England Journal of Medicine. 356(23):2388-2398.

Greville T. (1948). Mortality tables analyzed by cause of death // The Record. 37(76):283- 294.

Inter-university Consortium for Political and Social Research (2004). Multiple Cause of Death, 1968-1973. Ann Arbor, Mich.: Inter-university Consortium for Political and Social Research [distributor]. URL:http://webapp.icpsr.umich.edu/cocoon/ICPSRSTUDY/03905.xml.

(2007). Multiple Cause of Death Public Use Files, 2000-2002. Ann Arbor, Mich.: Inter-university Consortium for Political and Social Research [distributor]. URL: http://webapp.icpsr.umich.edu/cocoon/ICPSR-STUDY/04640.xml.

Jordan C. (1952). Life Contingencies. Chicago, Ill.: Society of Actuaries.

National Center for Health Statistics (1974). Vital Statistics of the United States, 1970: Life Tables. Vol. 2, Sec.5. Washington, DC: U.S. Government Printing Office.

Pearl R. (1922). The Biology of Death; Beinga Series of Lectures Delivered at the Lowell Institute in Boston in December 1920. Philadelphia; London: J.B. Lippincott Company.

Pollard J. (1982). The expectation of life and its relationship to mortality // Journal of Institute of Actuaries. 109 (Part II, No 442):225-240.

(1988). On the decomposition of changes in expectation of life and differentials in life expectancy // Demography. 25(2):265-276.

Pressat R. (1985). Contribution des écarts de mortalité par âge à la différence des vies moyennes // Population. 4-5:765-770.

Preston S., I. Elo, A. Foster, H. Fu. (1998). Reconstructing the size of the African American population by age and sex, 1930-1990 // Demography. 35(1):1-21. 
Preston S., P. Heuveline, M. Guillot (2001). Demography. Measuring and Modeling Population Processes. Malden, Massachusetts: Blackwell Publishers Inc., 2002 edition.

Preston S., N. Keyfitz, R. Schoen (1972). Causes of Death: Life Tables for National Populations. New York and London: Seminar Press.

Siegel J. (1974). Estimates of coverage of population by sex, race, and age in 1970 census // Demography. 11(1):1-23.

Siegel J., J. Passel (1976). New estimates of number of centenarians in United States // Journal of the American Statistical Association. 71(355):559-566.

Spiegelman, M. (1968). Introduction to Demography. Cambridge, Mass.: Harvard University Press.

United States (1972). 1970 census of population and housing: General Population Characteristics. Washington, DC.: U.S. Dept. of Commerce, Social and Economic Statistics Administration, Bureau of the Census.

United States Department of Health, Education, and Welfare (1968). United States Life Tables by Causes of Death: 1959-1961. (Life Tables: 1959-61, Volume 1, No. 6). Washington, D.C.: U.S. Government Printing Office.

U.S. Bureau of the Census (2005). Monthly postcensal resident population, by single year of age, sex, race, and Hispanic origin, 2000-2005. URL: http://www.census.gov/popest/national/asrh/2003_nat_res.html.

Vaupel J., V. Canudas-Romo (2002). Decomposing demographic change into direct vs. compositional components // Demographic Research. 7(1):1-14.

(2003). Decomposing change in life expectancy: a bouquet of formulas in honor of Nathan Keyfitz's 90th birthday // Demography. 40(2): 201- 216 